\title{
El inicio de la enfermería en México: Conflictos de poder y género, 1896-1904
}

\author{
The beginnings of nursing in Mexico: \\ Conflicts of power and gender, 1896-1904 \\ $O$ início da enfermagem no México: \\ Lutas de poder e de gênero, 1896-1904
}

A Elsa Malvido Miranda: En Memoriam (1941-2011)

Douglas C. Nance

MSN. Profesor-Investigador Asociado C, Tiempo Completo, Universidad de la Sierra Sur, Instituto de Investigación Sobre la Salud Pública.

Cómo citar este artículo en edición digital: Nance, D.C. (2018). El inicio de la enfermería en México: Conflictos de poder y género, 1896-1904. Cultura de los Cuidados (Edición digital), 22(50). Recuperado de http://dx.doi.org/10.14198/cuid.2018.50.08

Correspondencia: Universidad de la Sierra del Sur. C/Guillermo Rojas Mijangos s/n. Esq. Av. Universidad, col. Ciudad Universitaria. Miahuatlán de Porfirio Díaz, Oaxaca, México C.P. 70800.

Correo electrónico: biggato9@yahoo.com.

Recibido: 27/05/2017; Aceptado: 11/11/2017

Agradecimiento: Me gustaría expresar mi agradecimiento a la Dra. Oliva López Sánchez de la Facultad de Estudios Superiores Iztacala de la Universidad Nacional Autónoma de México para su revisión y sus comentarios, como siempre, astutos.

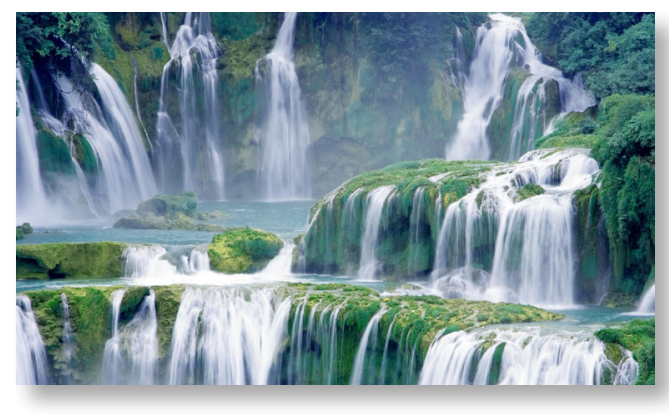

ABSTRACT

At the beginning of secular nursing in Mexico the career was involved in conflicts of power, role and gender with medicine.

Objective: This article explores the beginnings of nursing education in Mexico from a historical and epistemological perspective with a focus on power, role and gender.

Method: Primary sources have been used from the Historical Archive of the Ministry of Health of Mexico and Columbia University of New York and Mexican nursing journals, among others.
Results: The first directors-nurses in Mexico from 1896 to 1904, North American and British-American, were not submissive to doctors, which resulted in conflicts. The nursing school and the hospital have had a patriarchal environment where the "good" nurse has promoted the feminine values of piety, purity, submission and domesticity, resulting in the submission of the nurse and the exclusion of men.

Conclusions: Organized Mexican medicine only accepted those who know how to obey and submit. The result was the segregation of the nurse to a subordinate position. These conflicts and their results were definitive in the path that Mexican nursing would take.

Key words: History of nursing, gender role, nursing education, professional power

\section{RESUMEN}

En el inicio de la enfermería secular en México, la carrera se vio involucrada en conflictos 
de poder, rol y género con la medicina.

Objetivo: Este artículo explora los hechos que dieron inicio a la educación en enfermería en México con un perspectivo histórico y epistemológico con enfoque en poder, rol y genero.

Método: Se han empleado fuentes primarias del Archivo Histórica de la Secretaría de Salud de México y de la Universidad Columbia de Nueva York y revistas mexicanas de la enfermería, entre otros.

Resultados: Las primeras directores-profesores de enfermería en México de 1896 a 1904, norteamericanas y británico-estadounidenses, no fueron tan sumisas a los médicos, esto resultó en conflictos. La escuela de enfermería y el hospital ha tenido un ambiente patriarcal donde la enfermera "buena" ha promovido los valores femeninos de piedad, pureza, sumisión y domesticidad, resultando en la sumisión de la enfermera y la exclusión de los hombres.

Conclusiones: La medicina mexicana organizada solo se permiten aceptar a quienes saben cómo obedecer y ser sometidas. El resultado fue la segregación de la enfermera a una posición subordinada. Estos conflictos y sus resultados fueron definitivos en los caminos que tomaría la enfermería mexicana.

Palabras clave: Historia de la enfermería, rol de género, educación de enfermería, poder profesional

\section{RESUMO}

No início da enfermeira secular no México, a corrida foi envolvido em conflitos de poder, os papéis de gênero e da medicina.

Objetivo: Este artigo explora os acontecimentos que iniciaram o ensino da enfermagem no México, com uma perspectiva histórica e epistemológica com foco em papéis de poder e de gênero.
Método: Foram utilizadas fontes primárias do Arquivo Histórico do Ministério da Saúde do México e da Universidade de Columbia em Nova York e de revistas mexicanas de enfermagem, entre outros.

Resultados: A primeira faculdade diretores-enfermagem no México 1896-1904, americanos e britânicos-americanos não eram tão submissa aos médicos, isso resultou em conflitos. A escola de enfermagem e o hospital tinham um ambiente patriarcal, onde uma "bom" enfermeira promoveu valores femininos de piedade, pureza, submissão e domesticidade, resultando na apresentação da enfermeira e a exclusão dos homens.

Conclusões: Na medicina organizada mexicana só estão autorizados a aceitar aqueles que sabem como obedecer e ser apresentadas. $O$ resultado foi a segregação da enfermeira para uma posição subordinada. Estes conflitos e seus resultados foram definitivos nos caminhos que ele executaria na enfermagem do México.

Palavras-chave: história da enfermagem, papel do gênero, educação na enfermagem, de poder profissional.

\section{INTRODUCCIÓN}

En el inicio de la enfermería profesional en el siglo XIX, el trabajo de las enfermeras era el ambiente físico y emocional; sin embargo, el médico decidió cual era el trabajo "realmente importante" y de qué forma se había de hacer. Las enfermeras tomaron un papel subordinado, en la división de trabajo dominada por los hombres, lo que demuestra la importancia de las ideologías de género en las profesiones de cuidado. La idea del cuidado es una extensión del rol femenino, con las enfermeras de las clases obrera o media frente a los médicos de la 
clase alta, por lo tanto, estaba la subordinación de la enfermería a la medicina (Darbyshire, 1987). Estos conflictos desde el origen de la profesión han dejado huellas hasta el presente. En 1897 la enfermería no se consideraba una profesión en México. Los conflictos con la medicina organizada en relación con el papel de la enfermería inicio desde de la formación de la enfermería secular en México en este año. Los médicos querían mujeres "adiestradas" para llevar a cabo sus órdenes y para realizar las tareas arduas de cuidar, sin uso de juicio profesional, como el aseo personal del paciente y del entorno. Las primeras directores-profesores de enfermería en México fueron norte-americanas y británico-estadounidenses quienes tenían ideas distintas y vieron a la enfermería como una profesión complementaria, no idéntica, y no sumisa, a la de la medicina. Como la gran mayoría de las enfermeras eran mujeres, los roles de género tuvieron mucho que ver con estos conflictos. Estos conflictos no fueron exclusivos de México; los resultados fueron de una larga jornada sumisa para la enfermería hasta tiempos modernos.

Hoy en día, las responsabilidades de enfermería van mucho más allá de ser una sirvienta cuya responsabilidad principal es llevar a cabo las órdenes del médico. La evolución de la enfermería como una profesión es un resultado de la determinación y el trabajo duro de gente como las primeras enfermeras directoras-profesoras.

\section{OBJETIVO}

La historia de la enfermería durante el gobierno de Porfirio Díaz (1876-1911) todavía falta investigación. Algunas publicaciones erróneamente citan el inicio de la enfermería en México con el cambio de sede de la Escuela de Enfermería al Hospital General de México en 1907 (Salas Segura, Quesada Fox, \& Rubio Domínguez, 2010). Sin mencionar los años antes de 1907, ya que hay cronologías de eventos y fechas inexactas y falta de información sobre los inicios de la profesión (Pérez Loredo, 1986). El objetivo de este articulo es explora los hechos que dieron inicio a la educación en enfermería en México con un contexto histórico e epistemológico, con enfoque en los asuntos de poder, rol y genero.

\section{MÉTODO}

Para ejecutarlo se han empleado fuentes primarias del Archivo Histórica de la Secretaría de Salud de México y de la Universidad Columbia de Nueva York y revistas mexicanas de la enfermería, entre otros.

\section{RESULTADOS y DISCUSIÓN \\ Orígenes de la Enfermería Moderna}

En el siglo XIX han existido dos importantes modelos de formación de la enfermería que no fueron de órdenes religiosas católicas, el ejemplo de Kaiserwerth y el de Nightingale. El Hospital Kaiserwerth de Alemania formaron las enfermeras diaconisas protestantes, una réplica protestante al sistema monástica católica. Era modelo dependiente del médico donde las enfermeras debían cumplir las órdenes de éste. Con dependencia médico-técnica, disciplina semi-militar, y connotaciones religiosas, este modelo de ayudantes femeninas constituyen una imagen atractivo para los hombres de la naciente medicina científica-técnica. Florence Nightingale, fundadora de la enfermería moderna, inicio la profesionalización del cuidado en Gran Bretaña. La enfermería inicio como una opción profesional de contenido específico para investigar con la publicación de su libro "Notas Sobre la Enfermería" en 1859. Su modelo se centró en cuidar el enfermo, no la 


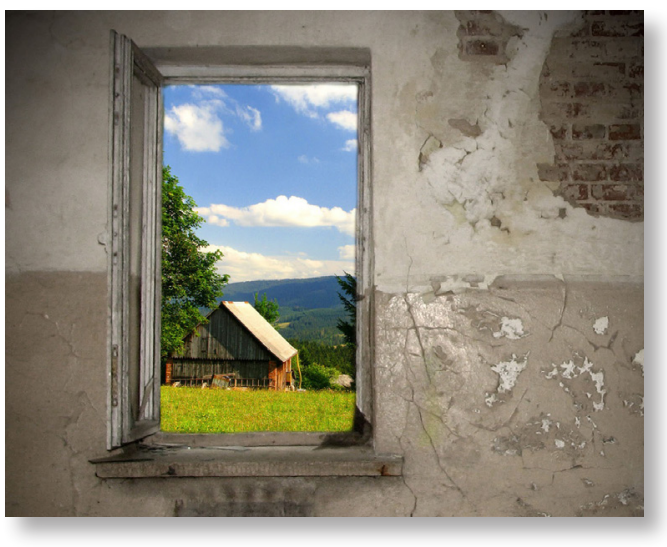

enfermedad. El éxito del modelo Nightingale ha tenido mucho que ver con el poder de Florence Nightingale de influir hombres poderosos y progresistas (y la Reina Victoria) con su brillantez intelectual, su lógica clara y su voluntad indomable. Su uso astuto de la prensa en la movilización de la opinión pública británica fue esencial para su éxito, construyendo así una imagen positiva de la enfermería. En México, las primeras líderes de la enfermería no tuvieron la ventaja de este poder de la imagen en la prensa, ni los nexos políticos con el poder, por lo que no fue posible lograr el mismo respeto o estatus en los años finales del siglo XIX y los inicios del siglo XX. En su principios de cuidar el enfermo y no la enfermedad, la Srta. Nightingale estableció que la enfermería se daba importancia al uso adecuado del aire fresco, la luz, el calor, la limpieza, la tranquilidad y de la administración de una dieta que ayudara a la fuerza vital del paciente. La investigación de las condiciones en que el paciente vive formó una parte esencial de la enfermería (Nightingale, [1859] 2008). Los primeros profesores de enfermería en México estuvieron de acuerdo con la Srta. Nightingale y con el famoso Dr. Kellogg, en que "la energía curativa no reside en el médico o el tratamiento, sino en una fuerza vital del paciente" (Kellogg, 1908) y esto se podría obtener con una dieta sana, con mucho granos, yogurt, aire fresco, ejercicio y pensamientos positivos. Esta actitud ante el paciente en la recuperación de su salud fue desde entonces distinta al pensamiento alopático con enfoque científico-tecnológico. El Dr. Kellogg fue un reconocido personaje debido a su hospital, spa, escuelas de enfermería y de medicina, y que además pasó a la historia con su hermano como los inventores de los corn flakes.

\section{Educación vs. Capacitación}

Selanders y Crane (2010) han denominado la lucha por la autonomía como "la lucha de la educación versus el aprendizaje de la alumna” o de educación vs. capacitación. La definición de la enfermería como profesión que requiere educación fue impulsada en 1893 en Chicago en la Exposición Colombina, la primera exposición importante donde las mujeres juegan un rol prominente. Una serie de Congresos fueron parte integral de la Exposición. Los Congresos proporcionaron una plataforma internacional para la discusión de temas sociales, y el Congreso de Hospitales, Dispensarios y Enfermería fue enfocada especialmente en asuntos de atención a la salud.

La enfermería, como una entidad educativa para la mujer, estaba en su segunda generación y muchos líderes de Europa y América del Norte participaron en el Congreso. Florence Nightingale envió un artículo importante, "Enfermería para los enfermos y enfermería para la salud", al Congreso. Con 73 años y mala de salud, ella no pudo asistir, pero su fama, su herencia, sus escritos y sus logros fueron su presencia. En el Congreso de Chicago, los influyentes y experimentados de enfermería describieron el futuro global de la enfermería para los próximos 50 años y los temas resonaron en las escuelas de enfermería (Bertuca, 1996). 
Los enfermeros norteamericanos y británicos, los primeros profesores de la enfermería profesional en México, fueron influenciados por las ideas que emanaban desde el Congreso. Estas ideas y la presencia aún viva de Florence Nightingale impregnaron las escuelas donde estos pioneros de la enfermería mexicana tuvieron su formación. Por el papel transcendente de los enfermeros norteamericanos en la formación de la enfermería mexicana en sus primeros años, el inicio de la enfermería profesional en México estaba muy vinculado con el del país vecino, los Estados Unidos de América.

Todos los profesores extranjeros (excepto las alemanas) tuvieron choques con el Dr. Eduardo Liceaga, director del proyecto de la nueva escuela de enfermería y líder de la medicina organizada mexicana, en la lucha por establecer a la enfermería con una visión de la salud distinto de la medicina alopática y con un proceso de educación, no de capacitación, en la formación de la Escuela de Enfermería en el capital de México entre 1896 y 1904. Esta lucha que se vivió en México fue un intento de la medicina para controlar y someter la enfermería. A través de los pocos documentos que sobreviven se ha recuperado algo de la lucha por la autonomía de la enfermería en México.

\section{La Expulsión de las Enfermeras en México}

La larga guerra civil que sostuvieron Liberales y Conservadores dio por resultado una invasión francesa, un archiduque austriaco como emperador fugaz y la restauración de la República en 1867. Las Leyes de Reforma, diseñadas para romper el poder económico y social del bastión conservador de la Iglesia Católica, incluyeron la supresión de las comunidades religiosas y la secularización de todos los hospitales y beneficencias que habían sido los proveedores de atención a los enfermos desde el siglo XVI.

Las Hermanas de la Caridad fueron la orden religiosa a la vanguardia de la enfermería. Consciente del daño que su abrupta expulsión ocasionaría, el presidente Benito Juárez exentó a esta orden de su supresión por ley (Ministro de Relaciones Exteriores y Gobernación, 1863). Pero con la muerte del Presidente Benemérito en 1872, Presidente Sebastián Lerdo de Tejada aplicó la ley sin importar de las consecuencias y expulsó a las Hermanas de la Caridad del país. En diciembre de 1874, a rodillas rezando, 410 Hermanas de la Caridad (355 de ellas mexicanas) fueron expulsados desde el puerto de Veracruz, por "una democracia incomprensiva y despótica” (Maza Brito, 1968).

Con la supresión de esta última orden religiosa, los hospitales de México se vieron sin enfermeras para cuidar a los enfermos. Capítulo esencial del plan para establecer un hospital general moderno fue la necesidad urgente para instituir una escuela de enfermería secular. A pesar del establecimiento de algunas escuelas de enfermería de protestantes diaconisas, muy pocas fueron las estudiantes se recibieron de diaconisas en México y se dejan pocas huellas. Las primeras fueron de la generación de 1903 en el Hospital Buen Samaritano de Guanajuato y el cual cerró en 1910. La revolución complica la situación de las diaconisas y la Constitución de 1917 fue explicita que la educación debería ser laica y este modelo de enfermeras protestantes no floreció en México (Malvido \& Hernández Cabrera, 2011).

\section{Planes para hospitales modernos pero faltan enfermeras}

El proyecto del Hospital General de México fue resultado de los ideales de la ciencia y el progreso a los que se aspiraba en el siglo XIX. 
A la cabeza del proyecto estaba el Dr. Eduardo Liceaga, presidente del Consejo Superior de Salubridad, director del Hospital de Maternidad e Infancia y médico personal del general Presidente Porfirio Díaz. El fue miembro de la élite científica que veía en "el Orden y el Progreso", máxima positivista de la época, los fundamentos para la construcción de un México moderno. Los impresionante proyectos de obra pública como el Hospital General de México y el Manicomio General de "La Castañeda” fueron pruebas concretas del orden y el progreso.

El Dr. Liceaga y sus colaboradores concibieron el proyecto para el nuevo hospital después de un largo viaje a los hospitales más avanzados en Europa y los Estados Unidos. A su regreso hicieron grandes planes; contaban con arquitectos y médicos, por supuesto con pacientes, pero faltaba un componente básico: debido a la expulsión de las monjas no había enfermeras en el país.

\section{La Búsqueda de una Directora-Profesora}

El Dr. Liceaga inició la búsqueda por una enfermera directora-profesora y escribió a sus colegas radicados en los Estados Unidos. Ya para aquellos años, este país contaba con una estructura formal de educación en enfermería, en universidades como Columbia y Johns Hopkins, que hubiera permitido establecer lazos institucionales sólidos y eficientes. No obstante, el Dr. Liceaga prefirió un medio personalista e informal. Dicha vía pudo tener sentido si sus intenciones eran controlar a la enfermería de una manera directa y personal. Como líder de la medicina organizada mexicana, el Dr. Liceaga obstaculizó el establecimiento de una enfermería independiente y profesionalizada desde el inicio.

En 1896, el artículo "Why are not Trained Nurses Sent to Mexico?” [¿Por qué no se en- vían enfermeras capacitadas a México?], se abunda en ejemplos sobre el lamentable estado del cuidado de los enfermos en el país y de la ausencia total de enfermeras capacitadas (Mignerez, 1896). La enfermera Margaret Simpson, en respuesta a este artículo, envió una carta a la Secretaría de Gobernación buscando empleo, pero ella no hablaba español (AHSS, 1896). Siguiendo con su búsqueda personal, el Dr. Liceaga continuó escribiendo a sus amigos médicos.

En julio de 1897, la Srta. Eva Cunningham, enfermera titulada de 22 años, producto de cuatro años de "capacitación y práctica rigurosa" en el Memorial Hospital de Brooklyn, Nueva York, envió su carta de interesada. Se describía a sí misma como "físicamente muy fuerte" y había vivido en la Ciudad de México con su familia, quien tenía negocios en la industria minera. Contratada como profesora por 100 pesos al mes, más hospedaje y comida, ella aceptó con la condición de que pudiera trasladarse a México para estudiar español, si estaba segura de iniciar en unos ocho a doce meses (AHSS, 1897).

El Secretario de Gobernación ordenaron a Dr. Liceaga para contratarla inmediatamente, "aprovechando el tiempo que falta para que se instale el hospital." Las expectativas eran que el hospital estuviera listo en diez meses, en realidad tardaría diez años en terminarse. No sabemos más de la carrera de la enfermera Cunningham, pero seguramente no siguió en su puesto como directora de una escuela inexistente.

\section{Primeros Directores- Profesores}

A finales de 1897, el Dr. Liceaga recibió a una pareja de enfermeros británicos-estadounidenses para dirigir la escuela. Los maridos Cooper, Alfred y Lillie, eran enfermeros 
titulados, egresados del Medical and Surgical Sanitarium Training School for Nurses de Dr. John Harvey Kellogg en Battle Creek, Michigan.

Los Cooper se graduaron el 1 de enero de 1896. En su currículo destacaban los cursos en higiene doméstica e individual, los usos médicos del agua, la electricidad y los masajes, también diversas materias de enfermería general, cirugía y obstetricia. El plan de estudios de la escuela de enfermería del Sanitarium fue igual que en las mejores escuelas, con temas adicionales de gimnasia médica y "un método compuesto fisiológico que comprendía la hidroterapia, fototerapia, termoterapia, electroterapia, mecanoterapia, la dietética, la cultura física, la terapia de aire frío y la formación en salud" (AHSS, 1897).

Alfred Cooper acompañó su solicitud de empleo con una carta firmada por José López Portillo y Rojas (notable autor y abuelo del futuro presidente de México), donde recomendaba ampliamente por haber cuidado su sobrino en una grave enfermedad y también por ser "una persona inteligente que había estudiado con el célebre Dr. Kellogg”. Ellos trabajaron en Guadalajara en la Casa de Salud de la Asociación Médico Misionera Benevolente del Séptimo Día y tras dos años, hablaban muy bien el español (AHSS, 1897).

Tanto los Cooper, como el Dr. Kellogg, eran Adventistas del Séptimo Día, iglesia cristiana que todavía está muy activa en cuestiones de salud y nutrición. En el Sanitarium del Dr. Kellogg se seguía una dieta vegetariana a base de granola y yogurt y se hacían ejercicios aeróbicos acompañados de música grabada y sesiones de risas organizadas para promover una actitud positiva. Además de la aplicación regular de enemas, se disponía de diversos tratamientos con modernos aparatos eléctricos.
El decreto Presidencial del 21 de abril de 1898 inauguraba la "Escuela Práctica y Gratuita de Enfermeros", adscrita al Hospital de Maternidad e Infancia, ubicado en la Calle de Revillagigedo de la Ciudad de México. Alfred y Lillie Cooper fueron contratados como profesores por un salario de 75 pesos al mes por un periodo de seis meses. La convocatoria decía lo siguiente:

Convocatoria para la Escuela Práctica y Gratuita de Enfermeros:

Deseando que esos enfermeros, sean personas de uno y otro sexo que tengan bastante cultura social, revelada por sus sentimientos humanitarios, su trato afable con los enfermos, sus hábitos de limpieza personal, y además la instrucción especial que se da a estos empleados en los grandes hospitales modernos, no se aceptaban alumnos que no reunían las siguientes condiciones:

Primera. Para inscribirse, se acreditara haber hecho la instrucción Primaria.

Segunda. Será una buena recomendación para las alumnas que se inscriban, haber cursado en la Escuela Normal o haber hecho estudios de Obstetricia.

Tercera. Para los del sexo masculino será una recomendación el haber hecho algunos cursos en la Escuela Preparatoria o haber desempeñado un empleo en las Oficinas Públicas a satisfacción de los superiores.

Cuarta. Los actuales enfermeros de los dos sexos, de los hospitales de la ciudad, podrán ser inscritos como alumnos, si acreditan con certificados del Director del Hospital al que sirven, su dedicación al trabajo, su manera afable para tratar a los enfermos y sus hábitos de aseo individual.

Quinta. Al inscribirse los alumnos, deberán presentar un certificado de buenas costumbres, (AHSS, 1898). 
La aceptación de hombres y mujeres, los altos niveles educativos necesarios para el ingreso de los alumnos y la atención a las buenas costumbres llamaron la atención.

Alfred y Lillie Cooper fueron los primeros profesores de enfermería en México. Su plan de estudio debe haber tenido bastante similitud con el del Dr. Kellogg. Los esposos Cooper partieron al término de su contrato y la Escuela y el Hospital siguieron siendo un proyecto sin cumplir. Es probable que la distinción entre la enfermería holística y la visión biomédica alopática de "orden y progreso" haya sido el motivo de su breve estancia.

La perspectiva de salud es un asunto de definición que marca la separación entre la enfermería y la medicina (Selanders \& Crane, 2010). La medicina da importancia primaria a la identificación de síntomas y su curación y la enfermería ha visto como temas de mayor importancia el cuidado, la promoción y la educación para la salud. La filosofía de los egresados de la escuela del Dr. Kellogg era "no curamos enfermedades, si no, pacientes, y recuperar sus condiciones normales y restaurar sus equilibrios vitales" (Kellogg, 1908). Nightingale (2008), afirmó que "la salud no es sólo para estar bien, sino para ser capaz de usar bien cada poder que tenemos", y "cuidar el enfermo, y no la enfermedad." Dudamos mucho que este enfoque haya sido del agrado del férreo positivista alopático Dr. Liceaga.

\section{La Búsqueda, Otra Vez}

La búsqueda continuó hasta 1900. A finales de ese año, Rose Crowder, una enfermera estadounidense de Missouri, llegó por el muy mejorado salario mensual de 500 pesos en oro, aprobado personalmente por el presidente Díaz. Titulada de la Eastern Illinois Hospital Training School for Nurses con formación especial en enfermedades mentales y un año atendiendo cirugías oculares en la Clínica Caritativa de Illinois para los Ojos y Oídos, Rose Crowder era una enfermera altamente capacitada. En su formación había tomado clases en dirección general, asepsia general y quirúrgica, obstetricia, administración de medicamentos, enemas, baños, masajes, enfermería general y cirugía. Llegó a México a los finales de noviembre de 1900 y se instaló en una casa de huéspedes, a la espera de sus aposentos en el Hospital de Maternidad e Infancia. Su plan de enseñanza incluye el siguiente:

Mi primera lección será impresionar sobre la necesidad de la limpieza a fondo en todo lo relacionado con la profesión, combinado con un orden y un sistema, y también con una buena disposición a todo, incluyendo la apariencia uniformada de cada enfermera. A continuación, cuando estos principios se hayan grabado en mis alumnas, comenzaré a trabajar con la atención al enfermo en cama.

Hay algunos principios importantes que enseñó a las alumnas desde los primeros días hasta su graduación, estos son: asepsia, bondad y la cortesía ante todo y para todos, alegría, y tanto en la formación como la práctica de la enfermería, la importancia de seguir las órdenes de los asesores médicos de forma explícita. Recordar que la enfermera no es un doctor (AHSS, 1900).

Pero no todo en sus planes arrojaría buenos resultados. Durante los dos años siguientes, la Directora pidió constantemente que su cuarto fuera completado y que se efectuara el pago de su salario. En diciembre de 1901 su salario se redujo drásticamente a 100 pesos. En marzo de 1902, seguía quejándose por la falta de una habitación y la falta de pago de sus comidas. Harta de todo, el 7 de mayo de 1902, anunció su renuncia. Inmediatamente el Dr. Liceaga 
ordenó que su habitación fuera terminada y le pidió que se quedara, pero ella se negó. Evitando todo responsabilidad, la explicación que dio el Doctor fue que "su resolución era inquebrantable porque no se avenía con algunos de los médicos del Hospital de Maternidad y se quejaba de la comida que se le daba" (AHSS, $\left.1902^{\mathrm{a}}\right)$.

Ante esos hechos, hubo que regresar a la búsqueda para encontrar una directora de la Escuela de Enfermería. En junio de 1902, se ofreció el puesto a la Srta. M. Bowman, enfermera titulada con 11 años de experiencia, dos de ellos en las Filipinas, por lo que hablaba español. Sin embargo, enojada y disgustada por las condiciones ofrecidas, ella rechazó la oferta con esta enérgica misiva.

Muy Señor mío:

Deseo informarle que no quiero aceptar el cargo de Enfermera Jefa de la Casa de Maternidad con las mismas condiciones que la señorita Crowder ha tenido... Ni por un momento consideraré la posición con el mismo sueldo que Usted ha estado pagando a la señorita Crowder. En cuanto a la comida, me moriría de hambre en una semana si yo tratara de vivir de las comidas que se sirven ahí. La habitación que la señorita Crowder ocupa podría ser utilizada si estuviera terminada correctamente. ... La Srta. Crowder ha sido más que considerada al soportar lo que ninguna otra enfermera estadounidense ha sufrido... Yo pasé cinco días en el hospital. Adjunto encontrará a cuenta de mis gastos. (AHSS, $\left.1902^{a}\right)$.

La enfermera Bowman ha soportado las condiciones en las Filipinas, en pleno estado de guerras coloniales desde 1899 al mismo año de 1902, con más de 200,000 de heridos y enfermos en las condiciones sanitarias problemáticas de una clima tropical. No sabemos cuáles fueron las condiciones insoportables en el pequeño Hospital pero suponemos que, en realidad, la comida no fue tan problemática como el Doctor.

\section{La Buena Adquisición y Nuevos Conflictos}

El mismo mes de junio de 1902, el médico Mariano Samaniego, de 70 años y buen observador de los encantos femeninos, reportó al Dr. Márquez:

Se encuentra en El Paso, Texas una Srta. Profesora titulada, de la que nada hay ya que desear, pues hasta posee el idioma español (es muy guapa), su trato es muy fino, su físico muy agradable, joven, pues cuenta sólo con 24 años, no le mando una fotografía, porque no tiene ninguna, solo posee una en grupo en que esta con sus compañeras de estudio. Después de larga práctica en el Hotel Dieu, fue examinada por un jurado de los Doctores más notables de la Cd., habiendo sustentado un examen muy lúcido. Francamente yo creo que se hará una buena adquisición. (AHSS, 1902b).

El Dr. Márquez le escribió a su vez al Dr. Liceaga: "en este momento me dirijo a El Paso, donde hay una enfermera titulada, que habla correctamente español, que desea ir a México y que por añadidura es muy guapa”, (AHSS, 1902b).

Esta guapa y bilingüe enfermera fue Rose Warden, quien egreso de la primera generación de la Escuela de Enfermería del Hospital Hotel Dieu en El Paso, Texas en 1902. Su padre John Warden había sido gerente de la Hacienda de Toluquilla en Omealco, Veracruz. De cual ironía, este hospital y escuela fue fundado y administrado por las mismas expulsadas Hermanas de la Caridad de San Vicente de Paúl. Por decisión médica colegiada se había encontrado a la directora perfecta, y muy guapa, para la Escuela de Enfermería. 
Recibiendo 50 pesos de oro para su pasaje, la enfermera Warden llegó a la Ciudad de México en julio de 1902, para encontrar que la habitación para la directora todavía no estaba lista. El Dr. Liceaga estaba sumamente complacido con ella y escribió: "La Srta. Warden está contenta y tiende a hacerse de amigos entre los que la rodean y les ha presentado una perspectiva muy halagüeña a las enfermeras, si éstas se empeñan en aprender." (AHSS, 1902b).

Pero poco a poco, los conflictos aparecieron. Como no fue parte de la enseñanza, en lugar de hacerlo, ella contrató gente de intendencia para ayudar a supervisar a las alumnas en sus dormitorios y contar sabanas. Su logro, y reto más importante fue el traslado de la Escuela desde el pequeño Hospital de Maternidad con apenas unas veinte pacientes, al Hospital de San Andrés con 600 pacientes, ubicado en la calle de Tacuba. Con este cambio se multiplicaron las oportunidades de aprendizaje para sus alumnos. A pesar de reconocer la sabiduría de la mudanza, el Dr. Liceaga no soportó que esa decisión, sin su participación, se tomara mediante una solicitud aprobada directamente por el Secretario de Gobernación.

\section{“No Sabe Ud. Hacerse Obedecer"}

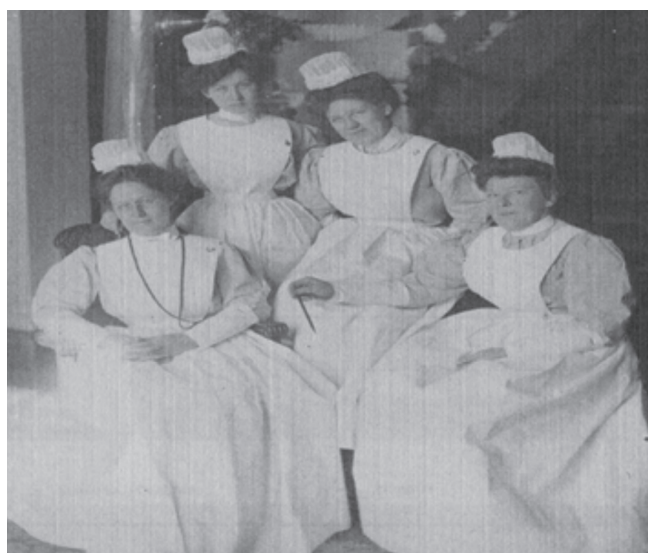

Imagen 1: Rose Warden, 1902 (arriba a la izquierda)
El 15 de marzo de 1904, el Doctor despidió a Rose Warden por insubordinación. En una larga carta, el Dr. Liceaga dejó en claro sus diferencias en la filosofía de la educación:

Mi intención era solamente preparar un cierto número de jóvenes que pudieran completar su educación más tarde, pero que fueran capaces de atender a los enfermos, de dar a los Médicos la reseñas que necesitaran, de hacer la administración de los pabellones. Para lograr esos objetos, era preciso en primer lugar, que completaran su educación social [énfasis del autor], (AHSS, 1904).

Liceaga se quejó de que la Directora Warden insistiera en la compra de uniformes para sus alumnas y materiales de curación. Le molestó también que hiciera cambios en las horas de las alumnas y de hacer cambios (no especificados) en sus relaciones con los médicos. El Doctor deja claras sus expectativas para la subordinación de la enfermera. La gran prueba de insubordinación fue la decisión de la Directora para cambiar la sede de la escuela a San Andrés, ante la decisión del Doctor de reducir el área clínica de la escuela solo al área de su consultorio (AHSS, 1904).

A pesar de que el Dr. Liceaga reconocía (y se quejaba) que la Directora Warden había mejorado el dormitorio de las enfermeras y el comedor, que instituyó el uso de uniformes (rayado de azul y blanco), insistió en materia de curación y que en general había mejorado la instrucción, el doctor externaba una queja porque la Directora no había prestado suficiente atención al registro adecuado de las sábanas limpias y sucias, y que no era lo suficientemente estricta en la disciplina. Y más allá de todo, "no sabe Ud. hacerse obedecer." No obstante, reiteraba que Rose Warden (Imagen 1) era "una enfermera muy inteligente, muy 
instruida, de muy buen carácter y muy agradable en sociedad, yo mismo la recomendaré por todas partes" (AHSS, 1904). La enfermera Warden renunció y salió el 3 de junio de 1904. Los conflictos de educación vs. capacitación y de la autonomía vs. la subordinación de la mujer-enfermera con el hombre-doctor no podían ser más claros.

Profesora y Directora de la Escuela de Enfermería, Rose Warden formó la primera generación de enfermeras tituladas en México y se instituyó el primer examen profesional de la carrera. En agosto de 1902, ingresaron 44 estudiantes, y en mayo de 1903, aprobaron 22 estudiantes, incluyendo enfermeros Félix Lejeva y Félix Tejada (AHSS, 1902, exp. 32).

Los conflictos entre el Dr. Liceaga y la enfermera Warden, atendieron a una diferenciación entonces irreconciliable en la promoción de la salud y la educación, imbuidos en complicaciones de género y poder que incidieron en el grado de autonomía e independencia del desarrollo de la enfermería profesional en México. Con la renuncia de la Directora Crowder y el despido de la Directora Warden, los médicos evidenciaron su control sobre las enfermeras y se impusieron sobre su formación profesional. La dominación al gremio de la enfermería por la medicina alopática quedó en manos masculinas mexicanas.

Rose Warden fue reemplazada por dos enfermeras alemanas, Gertrut Friedrich y Maude Dato, del Hospital de Ferrocarriles de México en Acámbaro, Guanajuato. Ambas fueron egresadas del Hospital Friedrichshain de Berlín, del modelo Kaiserwerth. Las alemanas al parecer no tuvieron problemas para seguir órdenes y aceptaron un salario bastante más baja, de 150 pesos de plata (AHSS, 1904).

Con la apertura del Hospital General de México, la Escuela de Enfermería cambió de sede y celebró la inauguración oficial de su nuevo hogar en 1907, y con el paso de los años, tuvo varias directoras de enfermería, estadounidenses y mexicanas. Dato y Friedrich continuaron de una forma $\mathrm{u}$ otra en el nuevo Hospital hasta por lo menos 1911. En 1911, una "supervisora alemana" puso muchos obstáculos para un grupo de enfermeras para salir a unirse a la Cruz Blanca Neutral para apoyar los heridos de la revolución (La Patria, 1911).

\section{DISCUSIÓN}

Cuando la escuela se pasó en 1911 a la sede de la Escuela de Medicina el control de los médicos fue total. El programa se redujo a unas pocas materias, solo suficientes para ser "capaces de atender a los enfermos, de dar a los Médicos la reseñas que necesitaran, de hacer la administración de los pabellones", para que "pudieran completar su educación más tarde", pero nunca fue completada. Sin embargo, la "educación social" fue bastante completa en términos del rol social de la enfermera.

La visión de la enfermería mostrada en 1893 en el Congreso sobre Hospitales, Dispensarios y Enfermería la posicionaba como una construcción social. Esa reunión, en el marco de una feria mundial, sirvió para movilizar a la enfermería hacia un estatus profesional basado en la percepción de su unicidad y su autonomía. En dicho Congreso, e igual que en los inicios de la enfermería mexicana, la salud fue vista no como la ausencia de enfermedad, sino como la maximización del potencial del paciente (Selanders \& Crane, 2010). En los inicios de la enfermería en México, los Cooper y "Las Rosas" (Crowder y Warden) ofrecieron una visión distinta del Dr. Liceaga sobre el concepto de salud y se luchó por su autonomía y el reconocimiento de su unicidad. 
Los enfermeros Félix Tejada (clase de 1902) y Félix Lejeva (clase de 1903) fueron titulados, pero poco después, los enfermeros mexicanos buscados por la Convocatoria de 1897 no fueron bienvenidos. Por 1907 el Dr. Liceaga prohibió a los hombres a entrar en la Escuela de Enfermería del Hospital General de México (Maza Brito, 1968).

Jovita Muñiz, alumna de Rose Warden, se unió a la Cruz Blanca Neutral en 1911 para apoyar los heridos de la revolución y le fue otorgada la Medalla de Plata de la Cruz Roja Internacional para su servicio (Nance, 2010). Adela Vásquez Schiaffino (clase de 1904) también ha prestado sus servicios de enfermera durante la Revolución en 1911. Enfermera, periodista, revolucionaria, poeta, novelista y maestra de idiomas, ella tampoco supo como “obedecer" (Nance, 2011).

\section{CONCLUSIONES}

Para 1907, excluyendo a los hombres, reduciendo la materia curricular de tres a dos años de estudio (Amézquita, 1955), y relegando a la enfermera a una posición subordinada y fácil de controlar; los médicos ganaron el control total de la educación, o mejor dicho, la capacitación, de la enfermería. Este fue parte esencial de la imposición de su práctica a la población y los mecanismos de exclusión, monopolización y control de otras profesiones sanitarias, que se consolidarían en el siglo XX (Coe, 1973). Este ansiosa preocupación sobre estatus, poder $\mathrm{y}$ rol habla mucho de los inseguridades del naciente profesión de la medicina alopática.

Welter (1966) describió el "culto a la verdadera feminidad" que abrazo cuatro valores de la mujer: piedad, pureza, sumisión y domesticidad. Intrínseca al valor de la domesticidad fue el deseo y la habilidad para cuidar la familia. Así, el cuidar fue visto como una

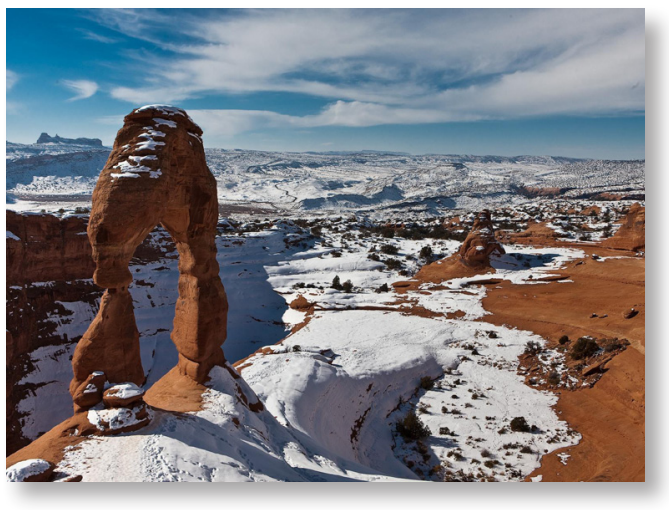

característica natural de ser mujer. Cuando la enfermería se cambia a un entidad definida, la necesidad fue la de conservar el ideal femenino y dar un cuerpo de habilidades especificas construido cuidadosamente a través del ambiente controlado del hospital. Los hospitales se quedan como un ambiente patriarcal donde la enfermera "buena" ha conservado los valores femeninos (Reverby, 1987). Este fue la educación social que el Dr. Liceaga pensaba que era más importante que la educación técnica o profesional.

Las enfermeras mexicanas estuvieron subordinadas al poder médico. La formación que se daba a las futuras enfermeras incluía, tanto en el aprendizaje como durante la práctica, la importancia de seguir cuidadosamente las prescripciones del médico y sus órdenes, recordándoles siempre que la enfermera no era ni podía ser médico, y en los expedientes de las alumnas se valoraba que estas fueran sumisas. Los estudios de enfermería fueron dependientes de la Escuela de Medicina, donde médicos eran los directores y maestros. La Asociación Mexicana de Enfermeras se crearía en 1947, y en 1948 la Escuela de Enfermería se separarían de la Escuela de Medicina, pero sin conseguir autonomía o autoridad, y tardaría hasta 1968 el inicio de la licenciatura (Carrillo, 1998).

A pesar de que ejercer la práctica del cuidado (la domesticidad) y posiblemente la pie- 
dad y la pureza, pero no sumisión, los Cooper, la Directora Crowder y la Directora Warden no fueron las enfermeras idóneas para el Dr. Liceaga. Las expectativas de la medicina mexicana organizada solo se permitían aceptara a quienes sabían cómo obedecer y permitir ser sometidas.

\section{BIBLIOGRAFÍA}

- Amézquita, S. (1955). Mis recuerdos de los primeros días de ingresar en el Hospital General. Revista de la Asociación Mexicana de Enfermeras, (3), 1.

- Carrillo, A.M. (1998). Profesiones sanitarias y lucha de poderes en el México del siglo XIX. Asclepio $(L) 2$, 149-168.

- Coe, R. M. (1973). Sociología de la medicina. Madrid: Alianza.

- Darbyshire, P. (1987). The burden of history. Nursing Times, 83(4), 32-34.

- Kellogg, J.H. (1908). The Battle Creek Sanitarium system: History, organization, methods. Battle Creek, MI: J.H. Kellogg.

- Maza Brito, M.E. (1968). Historia de la Enfermería en México. En E.M. Jamieson, M.F. Sewall, \& E.B. Suhrie (Eds.), Historia de la Enfermería (362-364). México, D.F.: Editorial Interamericana México.

- Malvido, E. \& Hernández Cabrera, M.E. (2011). Amor a Dios y amor al prójimo: Emociones básicas para la formación de las diaconisas metodistas protestantes y su expansión en el siglo XIX en México. En O. López Sánchez. La perdida del paraíso: El lugar de los emociones en la sociedad mexicana entre los siglos XIX y XX (309-325). México, D.F.: UNAM FESI

- Mignerez, A.U. (1896). Why are trained nurses not sent to Mexico? Trained Nurse Journal, 16(7), 356-358.

- Nance, D.C. (2010). Enfermeras del Hospital General de México a la Revolución. Revista de Enfermería del Instituto Mexicana de Seguro Social 18(2), 111-115.

- Nance, D.C. (2011). Adela Vásquez Schiaffino: una enfermera en la Revolución Mexicana. Revista de
Enfermería del Instituto Mexicano del Seguro Social, 19(1), 43-47.

- Nightingale, F. ([1859] 2008). Notes on nursing and other writings. New York, NY: Kaplan Publishing.

- Pérez Loredo, L. (1986). Efemérides de enfermería. México: Miguel Ángel Porrua.

- Reverby, S. (1987). Ordered to care: The dilemma of American nursing, 1850-1845. New York, NY: Cambridge University Press.

- Salas Segura, S., Quesada Fox, C. \& Rubio Domínguez, S. (2010). Origen y desarrollo de la carrera de enfermería y obstetricia en la Universidad Nacional Autónoma de México. México: UNAM/ENEO.

- Selanders, L.C. \& Crane, P. (2010). Florence Nightingale in absentia: nursing and the 1893 Columbian Exposition. Journal of Holistic Nursing, (28), 313-316.

- Welter, B. (1966). The cult of true womanhood: 18201860. American Quarterly, (18), 115-117.

\section{FUENTES}

- Archivo Histórico de la Secretaria de Salud (AHSS) (1896, 1897, 1898). F-SP, S-ED, Sc-HG, C-1, exp. 1-7, exp. 34.

- AHSS (1900). F-SP, S-ED, Sc-EE, C-1, exp. 8 (original en ingles, traducción por el autor).

- AHSS (1902a , 1902 b). F-SP, S-ED, Sc-EE, C-1, exp. 1-35, exp. 32.

- AHSS (1904). F-SP, S-ED, Sc-EE, C-1, exp. 4.

- La Patria. (1911, 17 Mayo) 5 enfermeras del Hospital General anoche debían haber partido para Cd. Juárez. La Patria. 1.

- Ministro de Relaciones Exteriores y Gobernación (1863). Ley que extinguió las comunidades de religiosas, artículo IX. México, D.F.: Diario Oficial de la Federación. 